\title{
Video-Assisted Thoracoscopic Surgery under O-Arm Navigation System Guidance for the Treatment of Thoracic Disk Herniations: Surgical Techniques and Early Clinical Results
}

\author{
Jung-Woo Hur ${ }^{1}$ jin-Sung Kim ${ }^{1} \quad$ Dong-Young Cho ${ }^{1}$ Jong-Mok Shin ${ }^{2}$ Jun-Ho Lee ${ }^{2}$ Sang-Ho Lee ${ }^{2}$ \\ ${ }^{1}$ Department of Neurosurgery, Seoul St. Mary's Hospital, Seoul, Korea \\ 2 Department of Neurosurgery, Wooridul Spine Hospital, Seoul, Korea \\ J Neurol Surg A 2014;75:415-421. \\ Address for correspondence Jin-Sung Kim, MD, Spine Center, \\ Department of Neurosurgery, Seoul St. Mary's Hospital, 222 Banpo \\ Daero, Seocho-gu, Seoul, 137-701, Korea \\ (e-mail: mddavidk@gmail.com; mddavidk@dreamwiz.com).
}

\begin{abstract}
This study describes the surgical technique and clinical results of video-assisted thoracoscopic surgery (VATS) assisted by an O-arm-based navigation system, used for the treatment of thoracic disk herniation (TDH). The trend toward the use of minimally invasive procedures with endoscopic visualization of the thoracic cavity in thoracic spine surgery has evolved. It is difficult to develop a new set of visuomotor skills unique to endoscopic procedures and understand the three-dimensional (3D) anatomy while performing a two-dimensional (2D) imaging procedure. Adding image guidance would have a positive impact on these procedures, making them safer and more precise. We report the results of 10 patients who underwent diskectomy for TDH using VATS assisted by an $\mathrm{O}$-arm-based navigation system and describe the surgical technique. The average duration of the symptoms was 2.8 years; average operation time, 326.9 minutes; and average additional time required for the image guidance surgery

Keywords

- thoracic disk herniation

- video-assisted thoracoscopic surgery

- navigation

- O-arm using the O-arm-based navigation, $\sim 29.4$ minutes. No complications occurred during the surgical procedure or the immediate postoperative period. The advantages of using navigational assistance during the surgical procedure include better visualization of the operative field, more accurate surgical planning, and optimization of the surgical approach involving the establishment of the correct drilling trajectory and safe decompression of the spinal cord, as well as the possibility of intraoperative control of bone resection.
\end{abstract}

\section{Introduction}

Thoracic disk herniation (TDH) is a rare condition, representing $<1 \%$ of all disk herniations in most series. ${ }^{1,2}$ The unusual symptoms of this condition may challenge early diagnosis. Routine use of magnetic resonance imaging (MRI) has increased the diagnostic rate of TDH. ${ }^{3,4}$ Anterior approaches to the thoracic spine have been established as the standard procedure for appropriate treatment of TDH because they permit direct visualization of the ventral spinal cord and

received

March 26, 2013

accepted after revision

December 10, 2013

published online

February 25, 2014

require less manipulation of the neural tissues in the spinal canal. $^{5-7}$ Thoracoscopic surgery of the spine has been reported in studies performed since the early 1990s. ${ }^{8,9}$

The development of surgical approaches to the spine has expanded the options for managing complex spinal pathologies in a minimally invasive manner. In the last decade, the extensive use of the microscope, endoscope, and two-dimensional (2D) or three-dimensional (3D) navigation systems has assisted surgeons in performing complex surgical procedures in a safer and less aggressive manner. ${ }^{6,9-13}$

(c) 2014 Georg Thieme Verlag KG Stuttgart · New York
DOI http://dx.doi.org/ 10.1055/s-0034-1368690. ISSN 2193-6315. 

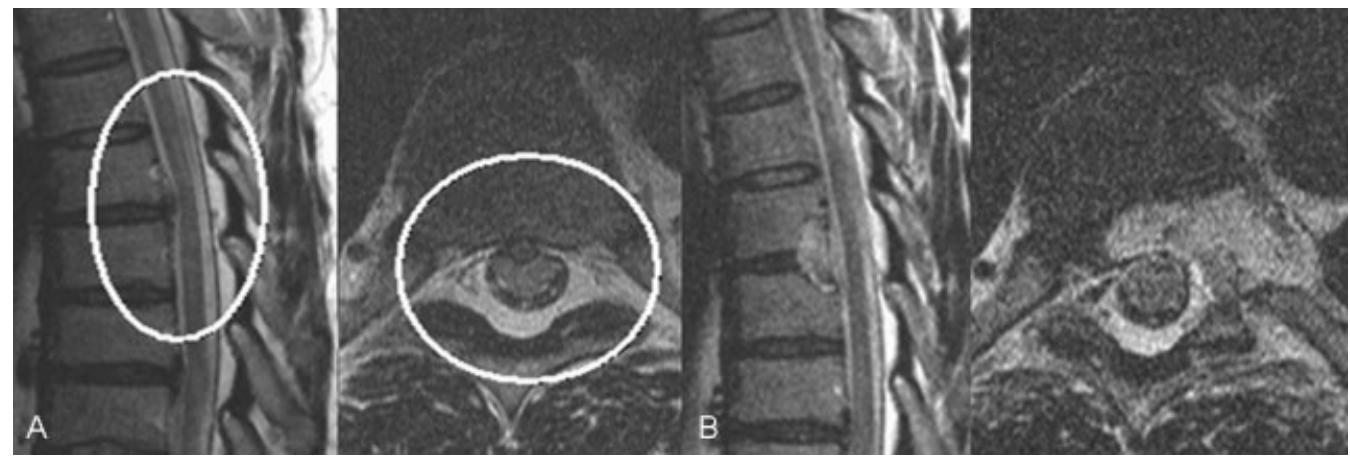

Fig. 1 (A) Preoperative sagittal and axial magnetic resonance imaging (MRI) showing disk protrusion causing compression at T6-T7. (B) Postoperative sagittal and axial MRI show a well-decompressed thoracic disk with a restoration of the thecal sac shape.

The trend toward the use of minimally invasive procedures with endoscopic visualization of the thoracic cavity in thoracic spine surgery has evolved. ${ }^{11,14,15}$ Both experienced and less experienced endoscopic spinal surgeons have often found it very difficult to develop a new set of visuomotor skills unique to endoscopic procedures and to understand 3D anatomy while performing a 2D imaging procedure. ${ }^{11} \mathrm{We}$ believe that introduction of an image guidance system would make these procedures safer and more precise. We report the results of 10 patients who underwent diskectomy for TDH using video-assisted thoracoscopic surgery (VATS) assisted by an O-arm-based navigation system and describe the surgical technique.

\section{Material and Methods}

This study describes the surgical technique of VATS assisted by O-arm navigation for the treatment of TDH and reports the clinical results of 10 patients who underwent surgery at our institution from September 2008 to April 2009 using this new technique. The inclusion criterion was the presence of soft TDH manifesting with myelopathy or radiculopathy despite conservative treatment. Patients with hard disk herniation, ossified posterior longitudinal ligaments, and/or concomitant moderate to severe bony spurs were excluded.

\section{Case 1}

A 45-year-old male patient presented with gait disturbance and a dull, aching radiating pain through the right and left buttocks and lower extremities; he had been experiencing these symptoms for the past 3 years. He also complained of impotence and numbness of the left flank and lateral calf. Physical examination revealed abnormally brisk deep tendon reflexes. The Babinski reflex and the ankle clonus signs were positive on both sides. MRI revealed cord compression caused by disk protrusion at the T6-T7 level ( - Fig. 1). The computed tomography (CT) scan showed soft disk herniation. TDH with myelopathy was diagnosed, and a thoracic diskectomy using VATS assisted by $\mathrm{O}$-arm-based navigation was performed. Three portals were created (-Fig. 2), and a partial diskectomy and decompression of the spinal canal was performed. The annulus was gently removed, thus exposing the posterior longitudinal ligament. The disk fragment was found in the epidural space, and the margin of the bony decompression was confirmed by the O-arm (-Fig. 3). After the operation, the patient experienced transient difficulty in voiding. He was discharged on day 17 after resolution of his voiding difficulty. Postoperative radiologic scans showed good decompression and no evidence of instability (-Fig. 1). At the most recent follow-up performed 12 months after the surgery, he could walk without any loss of balance. The impotence and

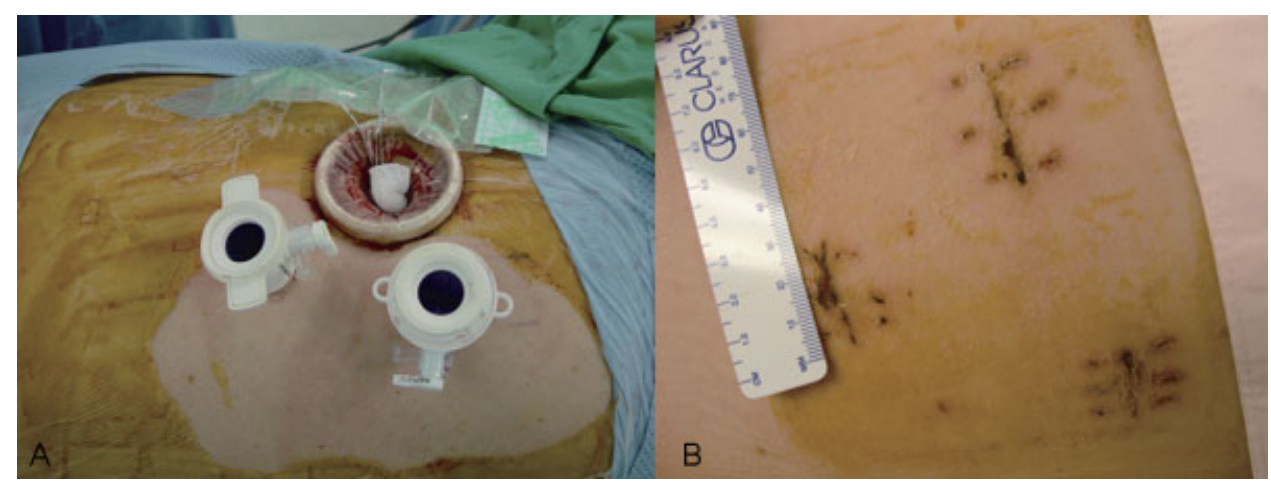

Fig. 2 (A) An intraoperative picture of the three portals created for the video-assisted thoracoscopic surgery showing the left side up position, with the cranial side on the right side of the image. The large portal is used for suction; the other two are working portals. (B) Postoperative wounds were $2 \mathrm{~cm}$ long. 


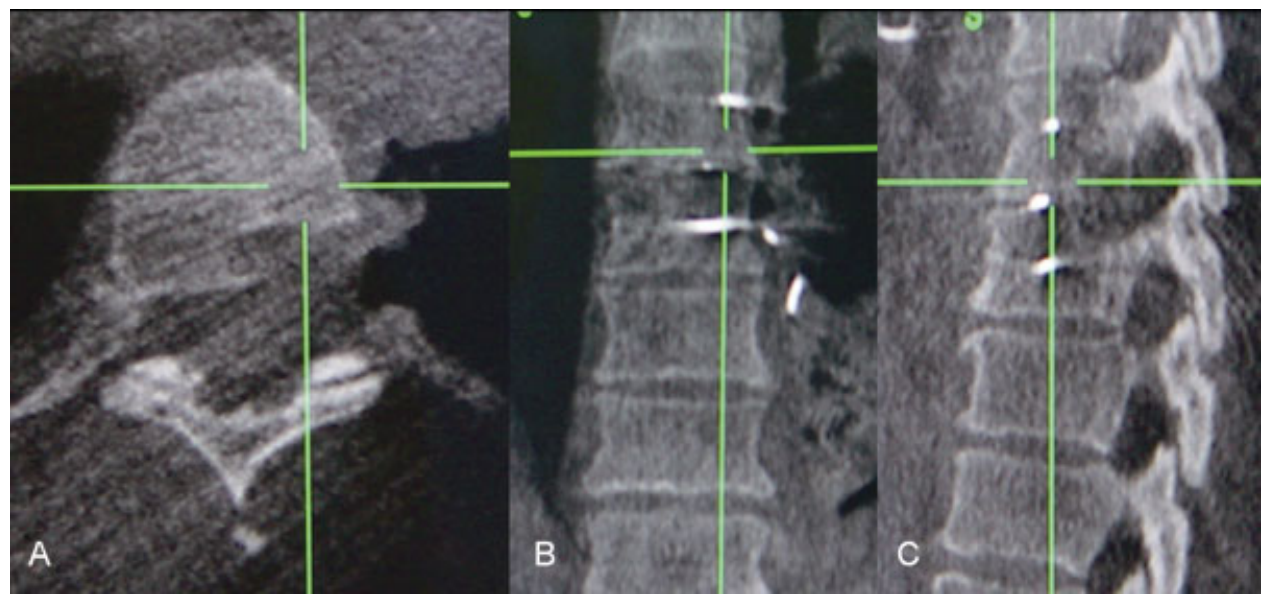

Fig. 3 The intraoperative O-arm (A) axial, (B) coronal, and (C) sagittal images enable the surgeon to determine the depth and the location of the drilling and show a decompressed bony margin.

radiating pain and numbness along both legs had greatly improved, although mild radiating pain persisted in the right buttock.

\section{Case 2}

A 63-year-old woman presented with gait disturbance, increased deep tendon reflexes, back pain, bilateral buttock pain, and radiating lower extremity pain. Radiologic investigations revealed a herniated disk at the T9-T10 level, with cord signal changes ( - Fig. 4), and degenerative spondylolisthesis with stenosis at levels L3-L5 and S1. We planned a twostage operation and performed a thoracic diskectomy first. Her postoperative MRI showed good decompression of the T9-T10 disk along with bony removal around the disk (-Fig. 4). At the 6-month follow-up, the radiating buttock pain showed improvement, and she was able to walk with better balance. However, the radiating pain in her lower extremity was not resolved. A subsequent lumbar lesion surgery was planned.

Eight other patients with similar symptoms underwent the same operation. - Table 1 presents additional patient information.

\section{Surgical Technique}

Under general double-lumen endotracheal anesthesia, the patient is placed in a left or right side up lateral decubitus position with nerve intramuscular monitoring. The first step in surgical navigation with the O-arm (Medtronic Sofamor Danek, Memphis, TN) involves a registration scan of the thoracic spine. The $\mathrm{O}$-arm is an intraoperative cone beam CT scan that provides 3D visualization. The data are reformatted by the workstation into coronal, axial, and sagittal 3D images of the spinal anatomy and transferred via a network connection to StealthStation navigation on a Treon system (Medtronic Sofamor Danek, Memphis, TN). The dynamic reference frame (DRF) is usually attached to the patient's iliac bone. However, in cases with upper thoracic level involvement, the DRF is attached to the more proximal operating table to reduce mismatching error. The coordinates of three to six intraoperatively identifiable anatomical landmarks of the level to be decompressed are obtained for use in the matching procedure during the surgery (-Fig. 5). The infrared camera is positioned facing the receiver, close to the operative field. This system is operated by a technician under the surgeon's guidance. The image guidance probe (IGP) is inserted through the portals in the chest wall to guide the surgical procedure to obtain a multiple planar or a 3D view.

An accuracy check, a crucial step in this procedure, is then performed to verify the quality of the matching. The surgeon has to determine whether the matching accuracy is acceptable for safe navigation by comparing the position of the instrument in the operative field with the displayed position

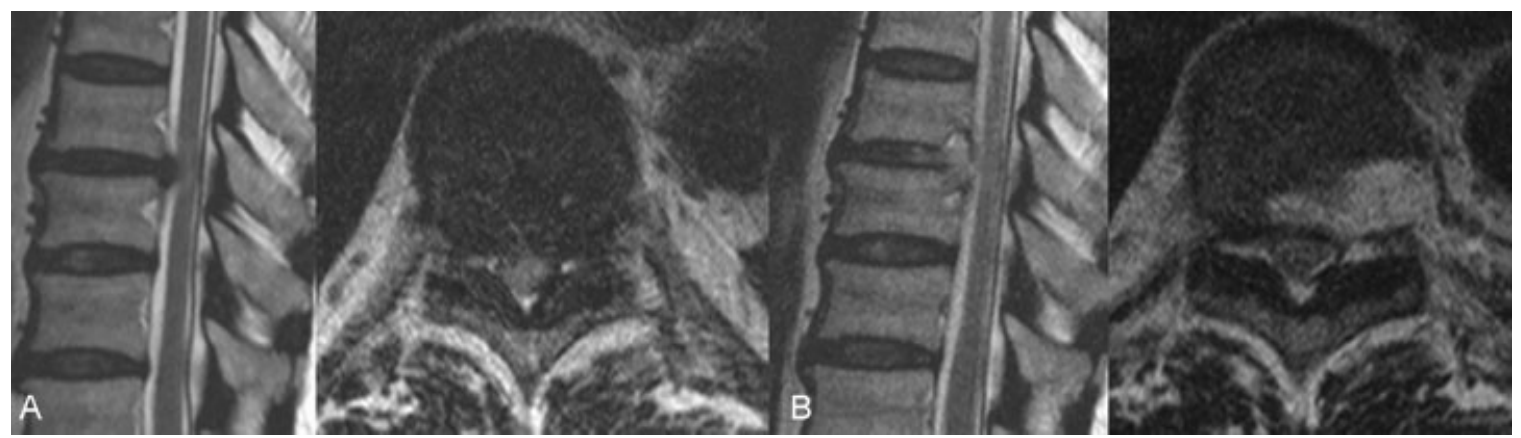

Fig. 4 (A) Preoperative sagittal and axial magnetic resonance (MR) images show disk protrusion at T9-T10 with cord signal changes. (B) Postoperative sagittal and axial MR images show a well-decompressed thoracic disk. 
Table 1 Demographics of video-assisted thoracoscopic surgical patients using intraoperative O-arm-based navigation ${ }^{\mathrm{a}}$

\begin{tabular}{|l|l|l|l|l|l|l|l|l|}
\hline Case no. & Sex & Age & $\begin{array}{c}\text { Thoracic } \\
\text { disk level }\end{array}$ & $\begin{array}{l}\text { Intraoperative } \\
\text { bleeding, } \mathbf{m L}\end{array}$ & $\begin{array}{c}\text { Total operation } \\
\text { time, min }\end{array}$ & $\begin{array}{c}\text { Time spent in } \\
\text { navigation and } \\
\text { O-arm, min }\end{array}$ & $\begin{array}{c}\text { Follow up } \\
\text { duration, mo }\end{array}$ & $\begin{array}{c}\text { Hospital } \\
\text { stay(day) }\end{array}$ \\
\hline 1 & $\mathrm{M}$ & 45 & $\mathrm{~T} 6-\mathrm{T} 7$ & 250 & 340 & 30 & 12 & 16 \\
\hline 2 & $\mathrm{~F}$ & 63 & $\mathrm{~T} 9-\mathrm{T} 10$ & 590 & 405 & 35 & 6 & 8 \\
\hline 3 & $\mathrm{M}$ & 56 & $\mathrm{~T} 10-\mathrm{T} 11$ & 420 & 330 & 20 & 5 & 5 \\
\hline 4 & $\mathrm{M}$ & 33 & $\mathrm{~T} 12-\mathrm{L} 1$ & 250 & 330 & 30 & 17 & 4 \\
\hline 5 & $\mathrm{~F}$ & 45 & $\mathrm{~T} 3-\mathrm{T} 4$ & 840 & 200 & 40 & 19 & 5 \\
\hline 6 & $\mathrm{M}$ & 54 & $\mathrm{~T} 6-\mathrm{T} 7$ & 310 & 360 & 20 & 12 & 13 \\
\hline 7 & $\mathrm{M}$ & 56 & $\mathrm{~T} 12-\mathrm{L} 1$ & 420 & 320 & 30 & 6 & 7 \\
\hline 8 & $\mathrm{M}$ & 77 & $\mathrm{~T} 11-\mathrm{T} 12$ & 450 & 330 & 30 & 13 & 6 \\
\hline 9 & $\mathrm{M}$ & 46 & $\mathrm{~T} 9-\mathrm{T} 10$ & 360 & 220 & 35 & 8 & 5 \\
\hline 10 & $\mathrm{~F}$ & 44 & T12-L1 & 220 & 310 & 25 & 11 & 7 \\
\hline
\end{tabular}

${ }^{\mathrm{a} A l l}$ diagnoses were thoracic disk with myelopathy.

of the instrument in the $\mathrm{CT}$ image on the monitor. If the accuracy is insufficient, the matching procedure is repeated.

After the localization of the disk level to be operated on using the IGP and the navigation system, three or four endoscopic portals are inserted for the VATS on the lateral chest. The first port is usually placed perpendicular to the main lesion. If the procedure involves the use of an endviewing 30- or 60-degree thoracoscope, the first port can be positioned above or below the perpendicular level. The ideal position of the port insertion is determined depending on the location of the target disk level identified on intraoperative $\mathrm{O}$ arm imaging and navigation in the StealthStation. After careful insertion of the first port to avoid violating pulmonary tissues, the second and third ports are inserted ventrally, making a triangular configuration on the ventral side. This layout allows the optic to be inserted through any port to obtain the optimum view at any time. Care is taken through- out the procedure to protect the collapsed lung by using an endoscopic fan retractor.

The first step in the thoracoscopic procedure is pleural exploration involving the identification of the major organs around the main lesion (e.g., the heart, great vessels, sympathetic chain, etc.), followed by opening of the parietal pleura at the appropriate spinal level using navigation. The second step involves the resection of $1.0 \mathrm{~cm}$ of the articulated rib. A partial diskectomy and partial vertebral body resection are performed using a high-speed drill in both cranial and caudal directions, until the normal dura margin is exposed. A Kerrison punch and pituitary forceps are then used to gently remove the ruptured disk fragments by cautious probing and removing the posterior longitudinal ligament, to achieve full decompression of the cord. Total decompression is verified when the pulsation of the spinal cord is visualized. At this point, if some bleeding is encountered, it can be managed by using Avitene (MedChem Products,
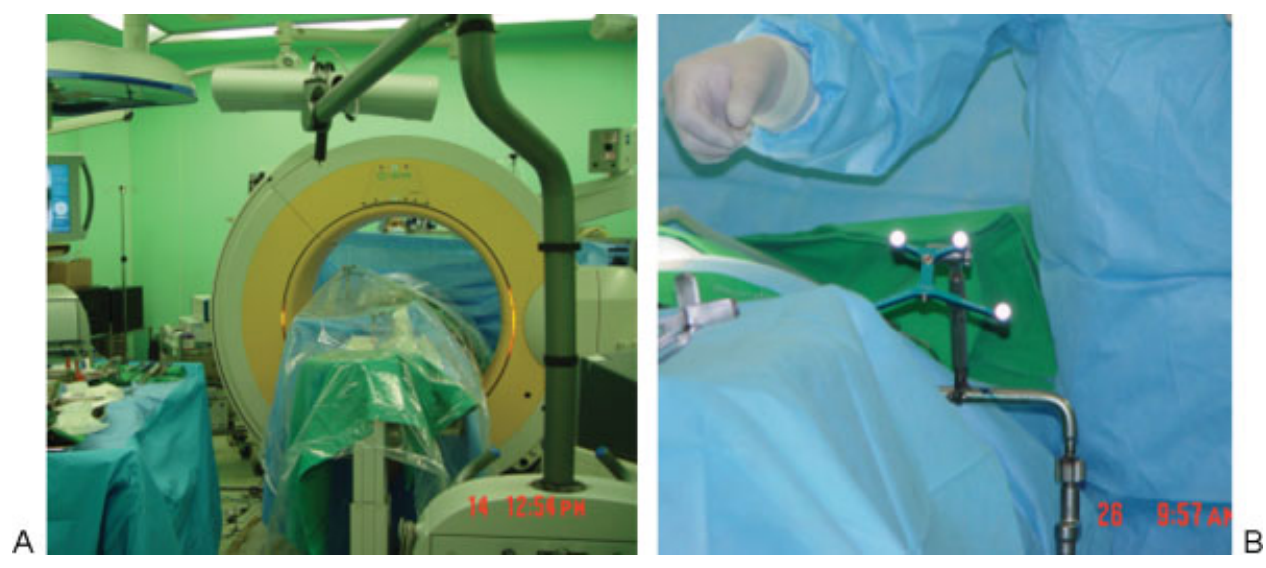

Fig. 5 (A) The operation room setting showing the navigation monitor, the dynamic reference frame, and the O-arm. (B) The dynamic reference frame on the table. 
Woburn, Massachusetts, United States), Gelfoam (Pfizer, Inc., New York, United States), and bone wax, but the use of bipolar coagulation is highly discouraged. The use of bipolar cauterization in this step may cause thermal injury to the spinal cord.

The O-arm enables precise determination of the margin of bone work and thus avoid injury to the spinal cord. Because the motion and view in thoracoscopy is limited compared with those in an open thoracotomy, the exact position of the drill is difficult to determine. The $\mathrm{O}$-arm enables the surgeons to precisely track surgical instruments in relation to their anatomy (i.e., accurately detect the depth and direction of the drill trajectory when approaching the target). After satisfactory decompression is achieved and the pulsation of the spinal cord is visualized, another scan of the thoracic spine using the $\mathrm{O}$-arm is performed to confirm the margin of the bone work and the efficacy of the decompression, giving the surgeon intraoperative feedback. If the margin of the decompression is found to be insufficient, the drilling and bone work can be extended immediately. - Table 2 shows the workflow and the time needed for each step.

Finally, with endoscopic guidance, a chest tube is placed through the posterior port, at which time the anesthesiologist reinflates the lung.

\section{Results}

Our patients included seven men and three women, with a mean age of 53.5 years (range: $35-77$ years). - Table 1 shows the distribution of the affected levels in the patients. Gait disturbance and back pain were the most common symptoms. Upper motor neuron abnormalities were present in all patients. The average duration of the symptoms was 2.8 years (range: $1-5$ years).

The average operation time was 326.9 minutes (range: 200-405 minutes), and the average blood loss during the surgical procedure was $441 \mathrm{~mL}$ (bleeding range: $250-840$ $\mathrm{mL}$ ). The average additional time required for the image guidance surgery using 0 -arm-based navigation was $\sim 29.4$ minutes (range: 20-40 minutes).

There were no complications during the surgical procedure or the immediate postoperative period. However, we experienced moderate intraoperative bleeding due to segmental vessel injury in one case (case 5 in - Table $\mathbf{1}$ ). Successful ligation was performed without any postoperative neurologic sequelae.

Table 2 O-arm workflow

\begin{tabular}{|l|l|}
\hline Procedures & Time, min \\
\hline Draping & 3 \\
\hline $\begin{array}{l}\text { Moving O-arm into surgical } \\
\text { field/ensure camera visibility }\end{array}$ & 6 \\
\hline Scanning & 1 \\
\hline Parking O-arm & 3 \\
\hline Uncovering sterile drape & 1 \\
\hline Ready to go & 14 \\
\hline
\end{tabular}

After the surgery, all patients' symptoms as well as walking improved. The mean follow-up period was 11.3 months (range: 5-19 months).

\section{Discussion}

With the advances in biotechnology and the development of specialized instruments such as improved microendoscopes, digital video equipment, and percutaneous systems, minimally invasive spinal surgery (MISS) has rapidly evolved and gained popularity in both spinal fusion and nonfusion surgery.

Recently, another new technique using computer-assisted surgery (CAS) for acquiring access in surgical decompression in spinal disease including cervical and thoracic disorders was shown to be useful ${ }^{12,13,16}$ because it enables real-time feedback to the surgeon about the anatomical location of the thoracic spine. This type of surgery can help the surgeon map anatomical distances and safe trajectories, thereby decreasing the risk of neural damage. Therefore CAS, in addition to being used in conventional open spinal surgeries, is also highly successful in other types of spinal surgery.

Therefore, we attempted to combine MISS with CAS to treat cervical disk herniation ${ }^{13}$ and TDH, and termed this procedure computer-assisted minimally invasive spinal surgery (CaMISS).

This introduction of CaMISS has not been well evaluated, and the surgical field in CaMISS is very narrow and limited. However, with the help of intraoperative O-arm and navigation in the StealthStation (Medtronic Sofamor Danek, Memphis, TN), the surgeon can determine the exact boundary of surgical decompression in a narrow operative field. ${ }^{17}$

Accurate intraoperative visualization of spinal anatomy is a crucial element in enabling thoracoscopic spine surgery. Precision is critical because of the unfamiliar thoracic anatomy such as the costochondral junction complex, narrow spinal canal, and proximity of bone margin to spinal cord. The success of the thoracoscopic approach depends on high-quality images so the surgeon can work within this complex environment. However, accurate knowledge of spinal anatomy totally depends on the surgeon's experience and the ability to visualize anatomical structures three-dimensionally.

Navigation in the StealthStation combines specialized surgical hardware with computer-assisted software that allows tracking of the location of an instrument in the surgical field, and a continuous update of this location within 3D planes (coronal, axial, and sagittal) is acquired through intraoperative $\mathrm{O}$-arm scanning. This virtual navigation allows the surgeon to navigate using multiple $\mathrm{CT}$ views simultaneously and avoid unwanted radiation exposure unlike conventional fluoroscopic navigation. Furthermore, with the aid of intraoperative CT, the surgeon can evaluate the spinal anatomy, correct the surgical path, and assess for correct instrumentation placed intraoperatively. Moreover, if an unfortunate change of the patient's position occurs, navigation re-registration is more feasible and fast with the $\mathrm{O}$-arm.

The accuracy of the image-guidance system may be affected by the technical accuracy of the system, the 
registration process conducted by the surgeon, the voxel size of the scan data, distortion of the image data, and intraoperative events that may result in errors. The O-arm allows both initial scanning and intraoperative registration of images. A major advantage of this system is that the surgeon can scan as many times as necessary to achieve adequate orientation during decompressive surgery or implantation. ${ }^{10-13,16}$

Several different approaches have been reported in the literature to treat TDH that can be classified into three categories: posterior, posterolateral, and anterior approaches. The first two approaches allow indirect decompression of the spinal cord and therefore carry the inadvertent cord injury risks. However, the anterior approach allows better visualization and direct decompression of the spinal cord, with the possible risks of pulmonary compromise. The choice of specific approach can be influenced by various factors including the disk consistency, the location in the canal, clinical presentation, the level of the pathologic lesion, and the surgeon's familiarity with the selected technique. There is no gold standard, and each approach has its own unique advantages and disadvantages. VATS is one of the important minimally invasive approaches to access anterior thoracic pathologies with several advantages including reduced postoperative pain and complication rates and faster recovery times. It is not yet familiar to most spine surgeons owing to its technically demanding performance, steep learning curve, and the need for special endoscopic instruments that are relatively expensive. Therefore, the posterior approach is still the more universally used procedure to treat TDH.

Many novel techniques have recently been introduced to overcome known disadvantages of the anterior approach including the minimally invasive transthoracic approach. ${ }^{18,19}$ With the help of a specially designed minimal invasive retractor system and combining it with thoracoscopy techniques, one could achieve safe access and excellent surgical views even without any rib resection. However, unfamiliar anatomy and unique characteristics of the thoracic spine with even smaller incisions may confound novice spine surgeons. Furthermore, the combination with thoracoscopy can aggravate the difficulties. The average spine surgeon requires additional training to acquire the skills to perform the procedure effectively.

A thoracoscopic procedure largely simulates an open thoracotomy procedure in decompressing the spinal canal. Since the early reports of thoracoscopic disk surgery, the indications of this technology have extended to thoracic vertebral reconstruction, as well as deformity correction. Using thoracoscopic techniques, anterior column reconstruction can be performed to provide effective loadbearing. In addition, thoracoscopic surgery provides small thin incisions without the need for rib resection or rib retractors, unlike other anterior approaches such as costotransversectomy or the transpedicular approach. Other advantages of this procedure include treatment of multisegmental abnormality through the same portals without the need for additional rib resection, excellent intra- operative visualization of the abnormality, direct anatomical access to the anterior structures of the thoracic spine, ${ }^{9}$ avoidance of diaphragm division, and significantly reduced chest wall injury. The VATS procedure is known to provide significant pain reduction, better cosmesis, lower perioperative morbidity, and an earlier return to normal activity. $2,6,8$

The disadvantages of the thoracoscopic spinal procedure are related to the dependence of the bidimensional endoscopic visualization on a video monitor; the working portals can significantly restrict the surgeon's movements in relation to the instruments, and the larger instruments require more dexterity. ${ }^{8,9}$ However, these disadvantages of thoracoscopic surgery can be decreased with the help of CaMISS based on Oarm navigation.

A lack of adequate training and a steep learning curve are the most common reasons for the limited use of thoracoscopic procedures. The absence of a 3D image of the surgical field and the lack of convenient guidance methods during endoscopic surgery can increase operating time; this does not encourage less experienced surgeons to use this technique. ${ }^{10}$

We believe the use of 3D image real-time guidance during VATS in thoracic spine would make the surgery safer and optimize spinal cord decompression. The biggest advantage of using surgical navigation is easier orientation in a limited operation field. Navigation enables a surgeon to find the target vertebral level, pedicle margin, and thecal sac more easily in a lateral decubitus position of the patient. Because both the motion and view of the thoracoscope is limited compared with those in an open thoracotomy, the exact margin removed by the drill is difficult to determine. If a protruded disk is on the midline, the diskectomy margin should go over the midline, and the opposite normal dural margin should be identified; this procedure is not easily performed through a thoracoscope. The O-arm allows the surgeons to check the depth of the working space and the direction of the trajectory in relation to the target. This provides important intraoperative feedback. Moreover, ideal portal placement is crucial in thoracoscopic surgeries because the surgeon's working view is greatly limited, and with poor portal placement, operating through the smaller portals with long surgical instruments can be difficult. We used IGP and the navigation system to decide the ideal portal position before the first port insertion depending on target disk location to overcome such difficulties. With the help of intraoperative $\mathrm{O}$-arm imaging and navigation in the StealthStation, we could avoid significant difficulty due to poor portal placement in all cases.

O-arm assisted navigation system has several great advantages over conventional spinal navigations. The image acquiring process to navigation set-up time is much shorter in the $\mathrm{O}$-arm-based system, and the patient does not have to change position or location, which greatly decreases accuracy because the spinal column is mobile. Furthermore, O-armassisted navigation systems do not need point-to point surface matching, like conventional spinal navigation, a factor that increases their use for further surgery without landmarks. 
As mentioned earlier, the VATS procedure requires an extremely steep learning curve for most spine surgeons to perform proper thoracoscopic techniques such as establishing proper orientation under the unfamiliar angled endoscopic views and operating through the smaller portals with long surgical instruments far from the target pathology. Furthermore, as with any other surgical techniques, there is a separate learning curve for using the O-armassisted navigation system. Surgeons have to practice the use of the planning modules to interpret CT image projections to identify anatomical landmarks and match them with the operation fields. Moreover, to identify the exact location of pathology, they have to learn to judge the intraoperative quality of the navigation accuracy. We had a little experience with conventional VATS or O-arm-assisted navigation technique previously, which increased the time required for disk removal as compared with others. We experienced a decrease in operative time from a mean of 360 minutes in the first three cases to a mean of 280 minutes in the last three cases. We hope further experience with thoracoscopic techniques will permit us to shorten the operative time.

The most common obstacles that surgeons encounter during VATS is intraoperative bleeding due to segmental or intercostal vessel damage. We experienced a case of segmental vessel injury during which intraoperative blood loss exceeded $800 \mathrm{~mL}$. Ligation of the segmental vessels is encouraged, although time consuming, to eliminate possibilities of catastrophic intraoperative or postoperative bleeding. To avoid such drawbacks during operation, it is important to identify and preserve the segmental vessels at the midportion of the vertebral body.

Although the surgical time may be longer than most conventional procedures and cost is a limiting factor in the wide use of this technique, the advantages of ensuring safety and determining the right trajectory, accompanied with appropriate bone decompression, compensate for these shortcomings. The surgeon is allowed real-time navigation of the unfamiliar thoracic anatomy, with the advantage of mapping anatomical distances and trajectories without fear of neurologic injury. O-arm-based navigation increases the accuracy of conventional VATS in a minimally invasive manner, leading to successful surgical outcomes; a longer-term follow-up is warranted.

\section{Conclusion}

Our combined use of O-arm-based navigation and thoracoscopic diskectomy was effective in assisting the surgeon simultaneously to perform and ensure a focal decompression of the spinal cord with better depth orientation. VATS assisted by $\mathrm{O}$-arm-based navigation is a viable, safe, effective, and minimally invasive option for treating TDH.

\section{References}

1 Arce CA, Dohrmann GJ. Thoracic disc herniation. Improved diagnosis with computed tomographic scanning and a review of the literature. Surg Neurol 1985;23(4):356-361

2 Choi KY, Eun SS, Lee SH, Lee HY. Percutaneous endoscopic thoracic discectomy; transforaminal approach. Minim Invasive Neurosurg 2010;53(1):25-28

3 Le Roux PD, Haglund MM, Harris AB. Thoracic disc disease: experience with the transpedicular approach in twenty consecutive patients. Neurosurgery 1993;33(1):58-66

4 Ross JS, Perez-Reyes N, Masaryk TJ, Bohlman H, Modic MT. Thoracic disk herniation: MR imaging. Radiology 1987;165(2): 511-515

5 Faciszewski T, Winter RB, Lonstein JE, Denis F, Johnson L. The surgical and medical perioperative complications of anterior spinal fusion surgery in the thoracic and lumbar spine in adults. A review of 1223 procedures. Spine 1995;20(14):1592-1599

6 Oskouian RJ Jr, Johnson JP, Regan JJ. Thoracoscopic microdiscectomy. Neurosurgery 2002;50(1):103-109

7 Sekhar LN, Jannetta PJ. Thoracic disc herniation: operative approaches and results. Neurosurgery 1983;12(3):303-305

8 Anand N, Regan JJ. Video-assisted thoracoscopic surgery for thoracic disc disease: classification and outcome study of 100 consecutive cases with a 2-year minimum follow-up period. Spine 2002;27(8):871-879

9 Johnson JP, Stokes JK, Oskouian RJ, Choi WW, King WA. Imageguided thoracoscopic spinal surgery: a merging of 2 technologies. Spine 2005;30(19):E572-E578

10 Assaker R, Cinquin P, Cotten A, Lejeune JP. Image-guided endoscopic spine surgery: Part I. A feasibility study. Spine 2001;26(15): 1705-1710

11 Assaker R, Reyns N, Pertruzon B, Lejeune JP. Image-guided endoscopic spine surgery: Part II: clinical applications. Spine 2001; 26(15):1711-1718

12 Bolger C, Wigfield C. Image-guided surgery: applications to the cervical and thoracic spine and a review of the first 120 procedures. J Neurosurg 2000;92(2, Suppl):175-180

13 Kim JS, Eun SS, Prada N, Choi G, Lee SH. Modified transcorporeal anterior cervical microforaminotomy assisted by $\mathrm{O}$-arm-based navigation: a technical case report. Eur Spine J 2011;20(Suppl 2): S147-S152

14 Burgos J, Rapariz JM, Gonzalez-Herranz P. Anterior endoscopic approach to the thoracolumbar spine. Spine 1998;23(22): 2427-2431

15 Rosenthal D. Endoscopic approaches to the thoracic spine. Eur Spine J 2000;9(Suppl 1):S8-S16

16 Haberland N, Ebmeier K, Grunewald JP, Hliscs R, Kalff RL. Incorporation of intraoperative computerized tomography in a newly developed spinal navigation technique. Comput Aided Surg 2000; $5(1): 18-27$

17 Schlenzka D, Laine T, Lund T. Computer-assisted spine surgery. Eur Spine J 2000;9(Suppl 1):S57-S64

18 Deviren V, Kuelling FA, Poulter G, Pekmezci M. Minimal invasive anterolateral transthoracic transpleural approach: a novel technique for thoracic disc herniation. A review of the literature, description of a new surgical technique and experience with first 12 consecutive patients. J Spinal Disord Tech 2011;24(5): E40-E48

19 Yanni DS, Connery C, Perin NI. Video-assisted thoracoscopic surgery combined with a tubular retractor system for minimally invasive thoracic discectomy. Neurosurgery 2011;68(1, Suppl Operative):138-143; discussion 143 